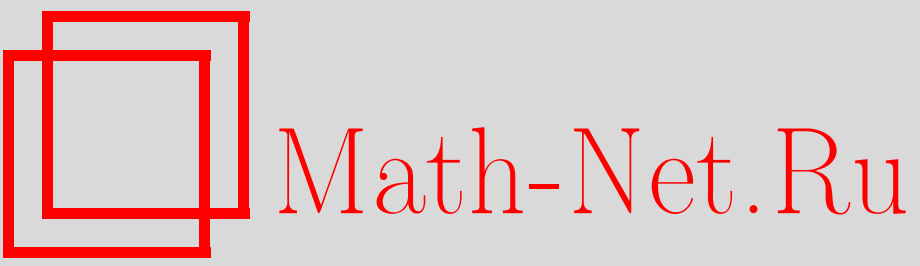

Б. В. Медведев, Гамильтониан и соотношения перестановки, ТМФ, 2000, том 122, номер 3, 325-333

DOI: https://doi.org/10.4213/tmf570

Использование Общероссийского математического портала Math-Net.Ru подразумевает, что вы прочитали и согласны с пользовательским соглашением

http://www.mathnet.ru/rus/agreement

Параметры загрузки:

IP: 54.172 .240 .79

26 апреля 2023 г., 15:59:33 
ТЕОРЕТИЧЕСКАЯ

И МАТЕМАТИЧЕСКАЯ

ФИЗИКА

Том 122, № 3

март, 2000

(C) 2000 г.

Б.В. Медведев

\section{ГАМИЛЬТОНИАН И СООТНОШЕНИЯ ПЕРЕСТАНОВКИ}

На конкретном примере демонстрируется, что задание перестановочных соотношений (в классике - скобок Пуассона) между основными динамическими величинами не менее существенно для спецификации рассматриваемой системы, чем задание гамильтониана.

1. В научном фольклоре стало обшепринятым отождествлять формулировку какойлибо - модельной или стремяшейся описывать природу - теории с заданием ее гамильтониана. При этом - вольно или невольно - оказьвается в тени роль перестановочных соотношений (в классическом описании - скобок Пуассона (CП)) вводимых динамических переменных. Такая непедантичность чревата возможностью досадных недоразумений, особенно когда, как то обычно бывает в теоретической физике, приходится заменять точное рассмотрение приближенным. Цель настоящей заметки состоит в том, чтобы наглядно продемонстрировать это обстоятельство на примере простейшей стохастической модели с двумя степенями свободы, введенной в научный оборот Матиняном и др. [1-3] в связи с исследованиями полей Янга-Миллса.

2. Эта система описывается гамильтонианом

$$
H=\frac{p_{x}^{2}+p_{y}^{2}+x^{2} y^{2}}{2}
$$

фигурируюшие в котором переменные $\left(x, p_{x}, y, p_{y}\right)$ удовлетворяют “обычным" перестановочным соотношениям или, в классическом описании, имеют “обычную” матрицу скобок Пуассона:

$$
(\mathrm{C \Pi})=\left(\begin{array}{cccc}
(x, x) & \left(x, p_{x}\right) & \ldots & \ldots \\
\left(p_{x}, x\right) & \left(p_{x}, p_{x}\right) & \ldots & \ldots \\
\ldots & \ldots & \ldots & \ldots \\
\ldots & \ldots & \ldots & \ldots
\end{array}\right)=\left(\begin{array}{cccc}
0 & -1 & 0 & 0 \\
1 & 0 & 0 & 0 \\
0 & 0 & 0 & -1 \\
0 & 0 & 1 & 0
\end{array}\right)
$$

Институт теоретической и экспериментальной физики, Москва, Россия 
которую мы, несколько условно, будем называть “диагональной”. Давно известно [4, 5], что эта система полностью стохастична, исключая множество меры нуль неустойчивых замкнутых траекторий. Гамильтоновы уравнения движения имеют вид

$$
\frac{d x}{d t}=p_{x}, \quad \frac{d p_{x}}{d t}=-x y^{2}, \quad \frac{d y}{d t}=p_{y}, \quad \frac{d p_{y}}{d t}=-x^{2} y .
$$

Поскольку система консервативна, то энергия $E=H$ сохраняется и доступная для движения часть конфигурационного пространства ограничивается парой равнобочных гипербол, так что $x^{2} y^{2} \leqslant 2 E=2 H$. Она естественно делится на неравноправные области: “арену", в которой $x^{2} \sim y^{2}<\sqrt{2 E}$, и четыре "рога", в которых либо $|y| \ll|x|$, либо $|x| \ll|y|$ (см. рисунок).

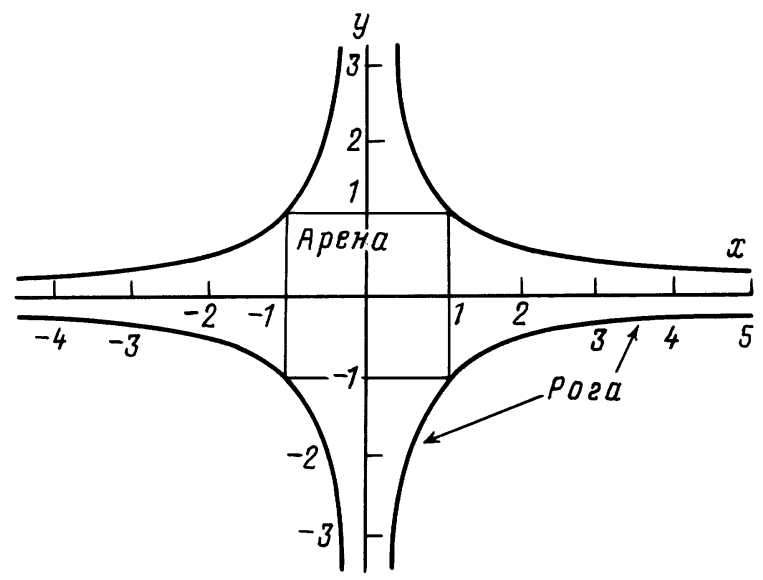

3. В каждом из рогов, будем считать для определенности, что в том, в котором $x>0,|y| \ll x$, можно построить приближсенне решение [6]

$$
\begin{aligned}
x & =x_{0}-I \frac{t^{2}}{2}, \quad y=\frac{\sqrt{2 I}}{\sqrt{x}} \cos \phi, \quad \phi=\int x d t=x_{0} t-\frac{I t^{3}}{6}, \\
p_{x}=-I t, \quad p_{y}=-\sqrt{2 I} \sqrt{x} \sin \phi, & \quad \phi \quad \\
\dot{x} & =-I t, \quad \dot{y}=-\sqrt{2 I} \sqrt{x} \sin \phi-\frac{y}{2 x} \dot{x}=-\sqrt{2 I} \sqrt{x} \sin \phi+\frac{\sqrt{2 I} I}{2 x \sqrt{x}} t \cos \phi \neq p_{y} .
\end{aligned}
$$

Оно точно удовлетворяет приближенным уравнениям

$$
\frac{d x}{d t}=p_{x}, \quad \frac{d p_{x}}{d t}=-\frac{x y^{2}}{2}-\frac{p_{y}^{2}}{2 x}, \quad \frac{d y}{d t}=p_{y}-\frac{y}{2 x} p_{x}, \quad \frac{d p_{y}}{d t}=-x^{2} y+\frac{p_{x} p_{y}}{2 x} .
$$

Чтобы это были обычные уравнения гамильтонова формализма, в котором переменные $\left(x, p_{x}, y, p_{y}\right)$ образуют канонический набор с матрицей скобок Пуассона (2), надо, чтобы эти уравнения имели бы форму

$$
\frac{d x}{d t}=\frac{\partial \widetilde{H}}{\partial p_{x}}, \quad \frac{d p_{x}}{d t}=-\frac{\partial \widetilde{H}}{\partial x}, \quad \frac{d y}{d t}=\frac{\partial \widetilde{H}}{\partial p_{y}}, \quad \frac{d p_{y}}{d t}=-\frac{\partial \widetilde{H}}{\partial y}
$$


с некоторой функцией Гамильтона $\widetilde{H}\left(x, p_{x}, y, p_{y}\right)$. Но чтобы последние уравнения были совместны, необходимо, чтобы выполнялись условия интегрируемости, например должно быть

$$
\frac{\partial}{\partial p_{y}} \frac{\partial \widetilde{H}}{\partial p_{x}}=\frac{\partial}{\partial p_{x}} \frac{\partial \widetilde{H}}{\partial p_{y}} .
$$

Между тем в системе (5), трактуемой как (6), мы получили бы

$$
\frac{\partial}{\partial p_{y}} \frac{\partial \widetilde{H}}{\partial p_{x}}=\frac{\partial}{\partial p_{y}} p_{x}=0
$$

в то время как

$$
\frac{\partial}{\partial p_{x}} \frac{\partial \widetilde{H}}{\partial p_{y}}=\frac{\partial}{\partial p_{x}}\left(p_{y}-\frac{y}{2 x} p_{x}\right)=-\frac{y}{2 x} \neq 0 \text { ! }
$$

Таким образом, уравнения (5) не могут быть уравнениями Гамильтона в форме (6) для какого бы то ни было $\widetilde{H}$ !

4. Эту неприятность удается устранить за счет того, что форма (6) не есть наиболее обшая форма гамильтоновых уравнений; такой является, как известно, форма

$$
\frac{d x}{d t}=(\widetilde{H}, x), \quad \frac{d p_{x}}{d t}=\left(\widetilde{H}, p_{x}\right), \quad \frac{d y}{d t}=(\widetilde{H}, y), \quad \frac{d p_{y}}{d t}=\left(\widetilde{H}, p_{y}\right),
$$

которая не будет совпадать с (6), если матрища (СП) скобок Пуассона не будет иметь специального “диагонального" вида (2). Чтобы использовать такую возможность, попробуем, например, изменить только равнявшиеся нулю СП $p_{x}$ с $y$ и $p_{y}$. Система (7) примет тогда вид

$$
\begin{array}{rlrl}
\dot{x} & = & \frac{\partial \widetilde{H}}{\partial p_{x}} & =p_{x}, \\
\dot{p}_{x} & =\frac{\partial \widetilde{H}}{\partial x}(-1) \quad+\frac{\partial \widetilde{H}}{\partial y}\left(y, p_{x}\right)+\frac{\partial \widetilde{H}}{\partial p_{y}}\left(p_{y}, p_{x}\right) & =-\frac{p_{y}^{2}}{2 x}-\frac{x y^{2}}{2} \\
\dot{y} & = & \frac{\partial \widetilde{H}}{\partial p_{x}}\left(p_{x}, y\right)+\frac{\partial \widetilde{H}}{\partial p_{y}} & \\
\dot{p}_{y}= & \frac{\partial \widetilde{H}}{\partial p_{x}}\left(p_{x}, p_{y}\right)+\frac{\partial \widetilde{H}}{\partial y}(-1) & & =\frac{p_{x} p_{y}}{2 x}-x^{2} y .
\end{array}
$$

Из уравнения (8а) следует, что $\widetilde{H}=p_{x}^{2} / 2+H^{\prime}\left(x, y, p_{y}\right)$. Далее, из (8в) с учетом (8a) получается, что

$$
p_{x}\left(p_{x}, y\right)+\frac{\partial H^{\prime}}{\partial p_{y}}=-\frac{y}{2 x} p_{x}+p_{y},
$$

откуда, разделяя члены, зависящие от $p_{x}$ и $p_{y}$, мы приходим к тому, что $\left(p_{x}, y\right)=-y / 2 x$ и $H^{\prime}=p_{y}^{2} / 2+H^{\prime \prime}(x, y)$. Используем теперь равенство (8г), которое тоже даст нам два уравнения: $p_{x}\left(p_{x}, p_{y}\right)=p_{x} p_{y} / 2 x$ и $\partial H^{\prime \prime} / \partial y=x^{2} y$, из которых получим, что $\left(p_{x}, p_{y}\right)=$ 
$p_{y} / 2 x$ и $H^{\prime \prime}=x^{2} y^{2} / 2+H^{\prime \prime \prime}(x)$. Таким образом, из (8а), (8в) и (8г) мы получаем для гамильтониана

$$
\widetilde{H}=\frac{p_{x}^{2}+p_{y}^{2}+x^{2} y^{2}}{2}+H^{\prime \prime \prime}(x) .
$$

Подставляя это выражение в оставшееся уравнение (8б), увидим, что оно удовлетворяется, если положить $H^{\prime \prime \prime}(x)=0$.

Итак, наша попытка придать системе (5) гамильтонову форму, допуская ненулевые значения для СП $\left(p_{x}, p_{y}\right)$ и $\left(p_{x}, y\right)$, увенчалась успехом: уравнения (5), которым удовлетворяют решения (4) приближенной задачи, могут быть приведены к гамильтоновой форме с "недиагональной" матрицей скобок Пуассона:

$$
(\mathrm{C} \Pi)=\left(\begin{array}{cccc}
0 & -1 & 0 & 0 \\
1 & 0 & -\frac{y}{2 x} & \frac{p_{y}}{2 x} \\
0 & \frac{y}{2 x} & 0 & -1 \\
0 & -\frac{p_{y}}{2 x} & 1 & 0
\end{array}\right) .
$$

(Тут следовало бы еше проверить, что так назначенные СП удовлетворяют тождествам Якоби; выкладка не составляет труда.) Самое же неожиданное состоит в том, что возникаюший для приближенного решения гамильтониан

$$
\widetilde{H}=\frac{p_{x}^{2}+p_{y}^{2}+x^{2} y^{2}}{2}=H
$$

есть просто гамильтониан точной модели Матиняна (1)!

ИТАК, из одного и того же гамильтониана, (т.е. из гамильтониана, для которого мы держим неизменной форму его зависимости от координат и импульсов) судя по тому, какие мы назначили правила перестановки, мы получаем:

ЛИБО полностью стохастическую модель Матиняна,

ЛИБО приближенную точно интегрируемую модель.

Задание перестановок (скобок Пуассона) играет в формулировке теории не менее кардинальную роль, чем задание гамильтониана.

5. В приближенной задаче переменные $\left(x, p_{x}, y, p_{y}\right)$ не образуют, как мы видим, канонического набора. Их можно, однако, выразить (с помошью неканонического преобразования) через другие, набор которых будет уже каноническим. Именно, если ввести новый комплект основных переменных $\left(x, \pi_{x}, y, p_{y}\right)$, положив

$$
\pi_{x}=p_{x}+\frac{y}{2 x} p_{y}, \quad p_{x}=\pi_{x}-\frac{y}{2 x} p_{y},
$$

то для них, как легко проверить, матрица СП будет иметь вид (2), т.е. они образуют канонический набор.

Но теперь гамильтониан, если выразить его через новые переменные, потеряет свою простую форму (10), принимая вид

$$
H_{\mathrm{app}}=\frac{1}{2}\left[\left(\pi_{x}-\frac{y}{2 x} p_{y}\right)^{2}+p_{y}^{2}+x^{2} y^{2}\right] .
$$


Гамильтониан (12) с перестановочными соотношениями (2) приводит для новых переменных к (гамильтоновым) уравнениям движения:

$$
\begin{array}{llrl}
\frac{d x}{d t} & =\pi_{x}-\frac{y}{2 x} p_{y}, & \frac{d \pi_{x}}{d t} & =-x y^{2}-\frac{y p_{y}}{2 x^{2}}\left(\pi_{x}-\frac{y}{2 x} p_{y}\right), \\
\frac{d y}{d t} & =p_{y}-\frac{y}{2 x}\left(\pi_{x}-\frac{y}{2 x} p_{y}\right), & \frac{d p_{y}}{d t} & =-x^{2} y+\frac{p_{y}}{2 x}\left(\pi_{x}-\frac{y}{2 x} p_{y}\right),
\end{array}
$$

которые точно интегрируются, приводя к решению

$$
\begin{gathered}
x=x_{0}-I \frac{t^{2}}{2}, \quad \pi_{x}=-I t-\frac{I}{x} \sin \phi \cos \phi, \\
y=\frac{\sqrt{2 I}}{\sqrt{x}} \cos \phi, \quad p_{y}=-\sqrt{2 I} \sqrt{x} \sin \phi, \\
\phi=\int x d t=x_{0} t-\frac{I t^{3}}{6},
\end{gathered}
$$

совпадаюшим, естественно, если вернуться в нем к старым переменным, с выписанным выше (4).

Итак, что касается приближенной системы, то мы видим, что ее равным образом можно описывать как диагональным по импульсам гамильтонианом (10) с "недиагональной” матрицей СП (9), так и переменными (11) с “диагональной” СП-матрицей (2), но зато с "недиагональным" гамильтонианом (12). При этом "значения" обоих гамильтонианов равны $H\left(x, p_{x}, y, p_{y}\right)=H_{\text {app }}\left(x, \pi_{x}, y, p_{y}\right)$, т.е. они получается один из другого (неканонической!) заменой переменных.

6. Что можно сказать об энергии приближенной системы? Сохраняется ли для приближенного решения точная энергия? Этот вопрос не совсем тривиален. В то время как сохраняющуюся энергию точного решения модели Матиняна можно было с равным правом выражать как через импульсы, так и через скорости

$$
E_{\mathrm{ex}}=\frac{p_{x}^{2}+p_{y}^{2}+x^{2} y^{2}}{2}=\frac{\dot{x}^{2}+\dot{y}^{2}+x^{2} y^{2}}{2}=\mathrm{const}
$$

при переводе этого выражения на язык приближенного решения возникает целый ряд альтернатив.

Через исходные импульсы $p_{x}, p_{y}$ - подставляя в точную энергию их приближенное выражение - мы получили бы

$$
E\left(x_{\mathrm{app}}, p_{x, \mathrm{app}}, \ldots\right)=\frac{p_{x}^{2}+p_{y}^{2}+x^{2} y^{2}}{2}=E=\mathrm{const} .
$$

Если же перейти к исправленным импульсам, для которых выполняются уравнения Гамильтона с диагональной СП-матрицей, то для сохраняющейся энергии $E_{\text {app }}$ возникнет уже знакомое нам выражение приближенной функции Гамильтона

$$
E_{\text {app }}\left(x, \pi_{\text {app }}, \ldots\right)=\frac{\left(\pi_{x}-\frac{y}{2 x} p_{y}\right)^{2}+p_{y}^{2}+x^{2} y^{2}}{2} .
$$


Но можно стать и на ту точку зрения, что приближенное решение определяет зависимость от времени только координат, а до импульсов и всей гамильтоновой структуры нам вообше нет дела. Тогда мы будем обязаны рассматривать и энергию как функцию координат и скоростей, получающихся из них простым дифференцированием по времени. В таком случае мы получили бы

$$
\begin{aligned}
& E_{\text {app }}(x, \dot{x}, \ldots)=\frac{\dot{x}^{2}+\dot{y}^{2}+x^{2} y^{2}}{2}= \\
& \quad=E\left(1-\frac{2 I \sqrt{2 E\left(1-\frac{x}{x_{0}}\right)}}{x} \sin \phi \cos \phi+\frac{I^{2} x_{0}}{x^{3}}\left(1-\frac{x}{x_{0}}\right) \cos ^{2} \phi\right) \neq \text { const } .
\end{aligned}
$$

Для приближенного решения, выраженного через координаты и скорости, полная энергия не сохраняется! Сохраняется же величина

$$
E_{\mathrm{app}}=\frac{\dot{x}^{2}+\left(\dot{y}+\frac{y}{2 x} \dot{x}\right)^{2}+x^{2} y^{2}}{2}
$$

- приближенная әнергия. Если мы посмотрим, как связана она с $E_{\text {app }}\left(x, \pi_{x}, \ldots\right)$ и с $E_{\text {ex }}\left(x_{\text {app }}, p_{x, \text { app }}, \ldots\right)$, то, естественно, убедимся, что численно все они совпадают.

7. В то время как исходная модель Матиняна выглядела чрезвычайно симметрично, в ней были диагональны и гамильтониан (1), и матрица СП (2), в обоих рассмотренных пока способах гамильтонова описания приближенной системы диагональность нарушалась: в одном диагональна функция Гамильтона (10), но недиагональна матрица СП (9), в другом СП-матрица диагональна, как в (2), но зато недиагональна кинетическая энергия в гамильтониане (12).

Хотелось бы найти такие переменные, в которых было бы диагонально и то, и другое. Для этого удобно отталкиваться от переменных $\left(x, p_{x}, y, p_{y}\right)$, в которых диагонален гамильтониан, и ввести еще одни импульсы

$$
P_{x}=f(x, y) p_{x}, \quad P_{y}=g(x, y) p_{y}
$$

отличающиеся от $p_{x}, p_{y}$ лиш множителями, зависящими только от координат. Такое преобразование не должно испортить диагональности кинетической энергии.

Чтобы добиться диагональности СП-матрицы в новых переменных, потребуем прежде всего, чтобы СП $\left(P_{x}, P_{y}\right)$ равнялась бы, как то для пары порядочных импульсов надлежит, нулю:

$$
\begin{aligned}
0 & =\left(P_{x}, P_{y}\right)=\left(f(x, y) p_{x}, g(x, y) p_{y}\right)= \\
& =\left(\frac{\partial f}{\partial x} p_{x}, f, \frac{\partial f}{\partial y} p_{x}, 0\right)\left(\begin{array}{cccc}
0 & -1 & 0 & 0 \\
1 & 0 & -\frac{y}{2 x} & \frac{p_{y}}{2 x} \\
0 & \frac{y}{2 x} & 0 & -1 \\
0 & -\frac{p_{y}}{2 x} & 1 & 0
\end{array}\right)\left(\begin{array}{c}
\frac{\partial g}{\partial x} p_{y} \\
0 \\
\frac{\partial g}{\partial y} p_{y} \\
g
\end{array}\right)= \\
& =\left(f \frac{\partial g}{\partial x}-f \frac{\partial g}{\partial y}+\frac{f g}{2 x}\right) p_{y}-\frac{\partial f}{\partial y} g p_{x}=0,
\end{aligned}
$$


что приводит к требованиям

$$
\frac{\partial f}{\partial y}=0 \quad \text { и } \quad \frac{\partial g}{\partial x}+\frac{1}{2 x} g-\frac{y}{2 x} \frac{\partial g}{\partial y}=0 .
$$

Обшим решением этой системы будет

$$
f(x, y)=f(x), \quad g(x, y)=\frac{1}{\sqrt{x}} h(\sqrt{x} y)
$$

с произвольными $f$ и $h$.

Введя новые импульсы, надо ввести, конечно, и новые координаты, которые мы будем считать какими-то функциями опять только старых координат

$$
X=X(x, y), \quad Y=Y(x, y)
$$

Потребуем канонических диагональных значений для остальных СП:

$$
\begin{aligned}
& \left(P_{X}, X\right)=f \frac{\partial X}{\partial x}-f \frac{y}{2 x} \frac{\partial X}{\partial y}=1 \\
& \left(P_{Y}, X\right)=\frac{h}{\sqrt{x} \frac{\partial X}{\partial y}}=0 \\
& \left(P_{X}, Y\right)=f \frac{\partial Y}{\partial x}-f \frac{y}{2 x} \frac{\partial Y}{\partial y}=0 \\
& \left(P_{Y}, Y\right)=\frac{h}{\sqrt{x}} \frac{\partial Y}{\partial y}=1 .
\end{aligned}
$$

Простые вычисления показывают, что обшим решением этих уравнений будет

$$
\begin{aligned}
X(x, y) & =X(x), \quad X(x) & =\int \frac{d x}{f(x)} \quad \text { или } \quad f(x) & =\frac{1}{\frac{d X}{d x}}=\frac{d x}{d X}, \\
Y(x, y) & =Y(\sqrt{x} y), & Y(\sqrt{x} y) & =\int \frac{d(\sqrt{x} y)}{h(\sqrt{x} y)} \quad \text { или } \quad h(\sqrt{x} y)=\frac{1}{\frac{d Y(\sqrt{x} y)}{d(\sqrt{x} y)}},
\end{aligned}
$$

где функции $f$ и $h$ остаются произвольными.

8. В этом состоит общий ответ на вопрос о том, когда преобразование (20), (24) приведет к каноническому набору. Для наглядности полезно разложить это преобразование на два этапа, на первом из которых простейшим выбором $f=1, h=1$ вводятся канонические переменные

$$
\begin{aligned}
& Q_{1}=x, \quad \frac{d Q_{1}}{d x}=1, \quad P_{1}=p_{x}=\pi_{x}-\frac{y}{2 x} p_{y}, \\
& Q_{2}=\sqrt{x} y, \quad \frac{d Q_{2}}{d(\sqrt{x} y)}=1, \quad P_{2}=\frac{1}{\sqrt{x}} p_{y}
\end{aligned}
$$


с обратными формулами

$$
x=Q_{1}, \quad p_{x}=P_{1}, \quad y=\frac{1}{\sqrt{Q_{1}}} Q_{2}, \quad p_{y}=\sqrt{Q_{1}} P_{2},
$$

и, поскольку в (неканонических!) $\left(p_{x}, x, p_{y}, y\right)$ приближенный гамильтониан равнялся просто

$$
H_{\mathrm{app}}=\frac{1}{2}\left(p_{x}^{2}+p_{y}^{2}+x^{2} y^{2}\right),
$$

то в (канонических!) $\left\{P_{1}, Q_{1}, P_{2}, Q_{2}\right\}$ он примет вид

$$
H_{\mathrm{app}}=\frac{P_{1}^{2}+Q_{1}\left(P_{2}^{2}+Q_{2}^{2}\right)}{2} .
$$

На втором этапе в рассматриваемых нами преобразованиях разрешенными остаются только сепаратные преобразования координат внутри каждой группы 1 и 2, не перемешиваюшие переменные разных групп:

$$
X=X\left(Q_{1}\right), \quad P_{X}=\frac{1}{\frac{d X}{d Q_{1}}} P_{1}, \quad Y=Y\left(Q_{2}\right), \quad P_{Y}=\frac{1}{\frac{d Y}{d Q_{2}}} P_{2} .
$$

После этого гамильтониан принимает вид

$$
H_{\mathrm{app}}=\frac{1}{2} \frac{1}{\left(\frac{d Q_{1}}{d X}\right)^{2}} P_{X}^{2}+\frac{Q_{1}}{2}\left(\frac{1}{\left(\frac{d Q_{2}}{d Y}\right)^{2}} P_{Y}^{2}+Q_{2}^{2}\right)
$$

откуда ясно, что преобразование $Y$-пары может только усложнить вид гамильтониана, в то время как преобразованием $X$-пары можно еще повысить его симметрию, например,

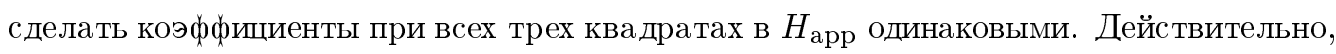
требуя

получаем

$$
\frac{1}{\left(\frac{d Q_{1}}{d X}\right)^{2}}=Q_{1}, \quad d X=Q_{1}^{\frac{1}{2}} d Q_{1}
$$

$$
X=\frac{2}{3} Q_{1}^{\frac{3}{2}}, \quad P_{X}=\frac{P_{1}}{\sqrt{Q_{1}}}, \quad Q_{1}=\left(\frac{3}{2} X\right)^{\frac{2}{3}}, \quad Q_{2}=Y,
$$

и гамильтониан приобретает форму

$$
H_{\text {app }}=\frac{1}{2}\left(\frac{3}{2} X\right)^{\frac{2}{3}}\left[P_{X}^{2}+P_{Y}^{2}+Y^{2}\right] .
$$

Окончательные же переменные выражаются через исходные как

$$
X=\frac{2}{3} x^{\frac{3}{2}}, \quad P_{X}=\frac{p_{x}}{\sqrt{x}}=\frac{\pi_{x}}{\sqrt{x}}-\frac{y}{2 x \sqrt{x}} p_{y}, \quad Y=\sqrt{x} y, \quad P_{Y}=\frac{1}{\sqrt{x}} p_{y}
$$

с обратными формулами

$$
x=\left(\frac{3}{2} X\right)^{\frac{2}{3}}, \quad p_{x}=\left(\frac{3}{2} X\right)^{\frac{1}{3}} P_{X}, \quad y=\frac{Y}{\left(\frac{3}{2} X\right)^{\frac{1}{3}}}, \quad p_{y}=\left(\frac{3}{2} X\right)^{\frac{1}{3}} P_{Y}
$$


9. Заметим в заключение, что наиболее удобной формой гамильтониана приближенной задачи является, по-видимому, все-таки форма (29), позволяюшая найти решение просто устным счетом "на пальцах". В самом деле, поскольку гамильтониан (29) зависит от $P_{2}$ и $Q_{2}$ только через

$$
I=\frac{P_{2}^{2}+Q_{2}^{2}}{2},
$$

то $I$ коммутирует с ним и, значит, сохраняется. А сумма квадратов чего постоянна, даже равна единице? - известно, синуса и косинуса. Значит, $Q_{2}$ и $P_{2}$ равны соответственно $\sqrt{2 I} \cos \phi(t)$ и $-\sqrt{2 I} \sin \phi(t)$ с некоторым $\phi(t)$. Далее, $I$ постоянно, поэтому движение $Q_{1}(t), P_{1}(t)$ есть тривиальное движение в постоянном поле. Наконец, вклад $I$ в гамильтониан пропорционален $x(t)$, поэтому $d \phi / d t=x$.

Благодарности. Автор признателен участникам семинаров в МИРАН и ИТЭФ за замечания, сделанные в процессе обсуждения докладов. В особенности мне хотелось бы поблагодарить И. Я. Арефьеву и П. Б. Медведева.

\section{Список литературы}

[1] Г. З. Басеян, С. Г. Матинян, Г. К. Саввиди. Письма в ЖЭТФ. 1979. Т. 29. С. 641.

[2] С. Г. Матинян, Г. К. Саввиди, Н. Г. Тер-Арутюнян-Саввиди. ЖЭТФ. 1981. Т. 80. C. 830 .

[3] С. Г. Матинян, Г. К. Саввиди, Н. Г. Тер-Арутюнян-Саввиди. Письма в ЖЭТФ. 1981. T. 34. C. 613

[4] Е. С. Николаевский, Л. Н. Шур. Пересечение сепаратрисс периодических траекторий и неинтегрируемость классических уравнений Янга-Миллса. Препринт ИТЭФ-3. М.: ИТЭФ, 1983.

[5] Б. И. Чириков, Д. Л. Шепелянский. ЯФ. 1982. Т. 36. № 6. С. 1563.

[6] Б. В. Медведев. ТМФ. 1984. Т. 60. С. 224; 1989. Т. 79. С. 404.

Поступила в редакцию 31.III.1999 г. 